\title{
Predictores ambientales De LA RIQUeZA DE ESPECIES DE PLANTAS DEL BOSQUe húMEDO de MONTAÑa de MÉXICO
}

\author{
Lauro López-Mata ${ }^{1,4}$, José Luis Villaseñor ${ }^{2}$, Gustavo Cruz-Cárdenas², \\ EnRIQue Ortiz ${ }^{2}$ y CARlos Ortiz-SOlorio ${ }^{3}$ \\ 'Posgrado en Botánica, Colegio de Postgraduados \\ 2Departamento de Botánica, Instituto de Biología, Universidad Nacional Autónoma de México \\ ${ }^{3}$ Posgrado en Edafología, Colegio de Postgraduados \\ ${ }^{4}$ Autor para la correspondencia: laurolopezmata@gmail.com y lauro@colpos.mx
}

\begin{abstract}
Resumen: El bosque húmedo de montaña, también conocido como bosque mesófilo de montaña o cloud forest, está definido y limitado por la presencia persistente de nubes y neblina que, además de la lluvia, capturan las gotas de agua que se condensan sobre la vegetación. Se reconoce ampliamente que la humedad atmosférica es el principal factor ambiental que promueve la persistencia y distribución de este tipo de vegetación en el mundo. Sin embargo, se carecen de análisis correlativos entre riqueza de especies del bosque húmedo de montaña y los factores ambientales a escalas amplias. Es de suponer que tal riqueza tiene su base en la hipótesis correlativa con variables ambientales contemporáneas, principalmente con la tríada altitud-clima-suelo. El objetivo fue analizar e identificar que variables ambientales tienen asociación significativa y cuáles son las más importantes en la predicción de la riqueza de especies del bosque húmedo de montaña. Los sitios con bosque húmedo de montaña se ubicaron en 61 celdas de $1^{\circ}$ latitud $\times 1^{\circ}$ longitud. Mediante sistemas de información geográfica se generaron capas ambientales que incluyeron variables bioclimáticas, de estacionalidad climática, un modelo digital de elevación y variables edáficas. Los resultados indican que $58 \%$ de la variación en la riqueza de especies del bosque húmedo de montaña se relaciona con cinco variables: la precipitación pluvial de los meses húmedos del año, la altitud, la evapotranspiración real anual, la estacionalidad de la precipitación y el carbono orgánico en los suelos. En el bosque húmedo de montaña de México existe una asociación consistente entre la riqueza de especies y las expresiones categóricas de la relación agua-energía.
\end{abstract}

Palabras clave: altitud, biodiversidad, bosque mesófilo de montaña, distribución, evapotranspiración.

\begin{abstract}
The humid mountain forest also known as cloud forest or bosque mesófilo de montaña, is defined and delimited by the persistent occurrence of clouds and fog, which in addition to rain capture water drops that condensate over vegetation. It is well known that atmospheric humidity is the main factor promoting its persistence and distribution all over the world. However, it is evident the lack of correlative analyses between species richness of the humid mountain forest and the environmental factors to large scales. It is supposed that such richness is based on the correlative hypothesis with contemporaneous environmental variables, particularly the triage altitude-climate-soil. The aim of this paper is to analyze and identify what environmental variables have significant association with species richness of the humid mountain forest and which of them are the most important predictors. The sites with humid mountain forest in Mexico were located in 61 grid cells each $1^{\circ}$ latitude and longitude. By using a Geographical Information System, several environmental layers were generated, including bioclimatic and climatic seasonality variables, a digital elevation model, and edaphic variables. The results indicate that $58 \%$ of species richness variation in the humid mountain forest is explained with five variables: the rainfall of humid months of the year, the altitude, the real annual evapotranspiration, the seasonal rainfall, and the organic carbon contents in soil. In the humid mountain forest of Mexico a consistent association exists between the species richness and the categorical expressions of the relationship water-energy.
\end{abstract}

Key words: biodiversity, cloud forest, distribution, elevation, evapotranspiration.

$\mathbf{E}_{\mathrm{s}}$ bosque húmedo de montaña (BHM), más conocido en México como bosque mesófilo de montaña, caracteriza a los ecosistemas montañosos donde las masas de aire de alta humedad alcanzan el punto de saturación que resulta en ambientes de elevada humedad atmosférica. Este bosque se distribuye en una franja altitudinal relativamente angosta que experimenta la presencia de nubes y neblina (Hamilton et al., 1995), abundante precipitación, elevada condensa- 
ción de agua sobre la vegetación y baja tasa de pérdida por evapotranspiración (Bruijnzeel et al., 1993). Esta condición ambiental genera influencias en los ámbitos hidrológicos, ecológicos, fisiológicos, estructurales, de composición florística, de fisonomía del bosque y de propiedades de los suelos (Huber, 1986; Stadtmuller, 1987; Bruijnzeel et al., 1993). Vogelman (1973) sostiene que las temperaturas moderadas y la elevada humedad atmosférica son las principales determinantes ambientales de este tipo de vegetación. La elevada incidencia de nubosidad incrementa la incidencia de luz solar difusa que provoca una disminución de la temperatura y el mantenimiento de una humedad atmosférica elevada; tanto la baja radiación solar directa y la elevada humedad atmosférica reducen el déficit de presión de vapor de agua. La humedad atmosférica es un factor ambiental de primordial importancia para la persistencia y distribución de este tipo de vegetación en el mundo (Churchill et al., 1995; Hamilton et al., 1995; Kappelle y Brown, 2001). Velázquez et al. (2000) añaden que el establecimiento del BHM requiere, además de una humedad relativa alta, un ambiente montañoso, topografía heterogénea, suelos con una capa profunda de hojarasca y clima templado.

En México, los climas donde prevalece el BHM son los más cálidos entre los climas templados, con temperatura media anual entre 12 y $23{ }^{\circ} \mathrm{C}$ y precipitación entre 1,000 y $5,800 \mathrm{~mm}$ anuales, pero generalmente es alrededor de 2,000 $\mathrm{mm}$ anuales. Las lluvias se presentan generalmente en el verano y decrecen de diciembre a mayo. Los periodos secos son cortos y poco intensos, ocurriendo de cero a cuatro meses del año.

La distribución del BHM en México es discontinua, semejando un archipiélago de parches de bosque (VázquezGarcía, 1995), sobre las principales cadenas montañosas y localmente en fragmentos de vegetación entre 1,500 y 2,500 $\mathrm{m}$ de altitud. Se distribuye sobre la Sierra Madre Occidental desde Sonora hasta Michoacán; en la Sierra Madre Oriental desde el sur de Tamaulipas hasta el centro de Veracruz; a lo largo de la Faja Volcánica Transmexicana; en la Sierra Madre del Sur en Guerrero y Oaxaca; en la Sierra Norte de Oaxaca y en la Sierra Madre de Chiapas (Figura 1). Este bioma incluye a un conjunto amplio de asociaciones heterogéneas que varían geográficamente en la composición florística, estructura, estado de conservación y grado de perturbación al que están siendo o han estado sometidos (Rzedowski, 1996; Challenger, 1998; Ramírez-Marcial et al., 2001; Luna-Vega et al., 2006).

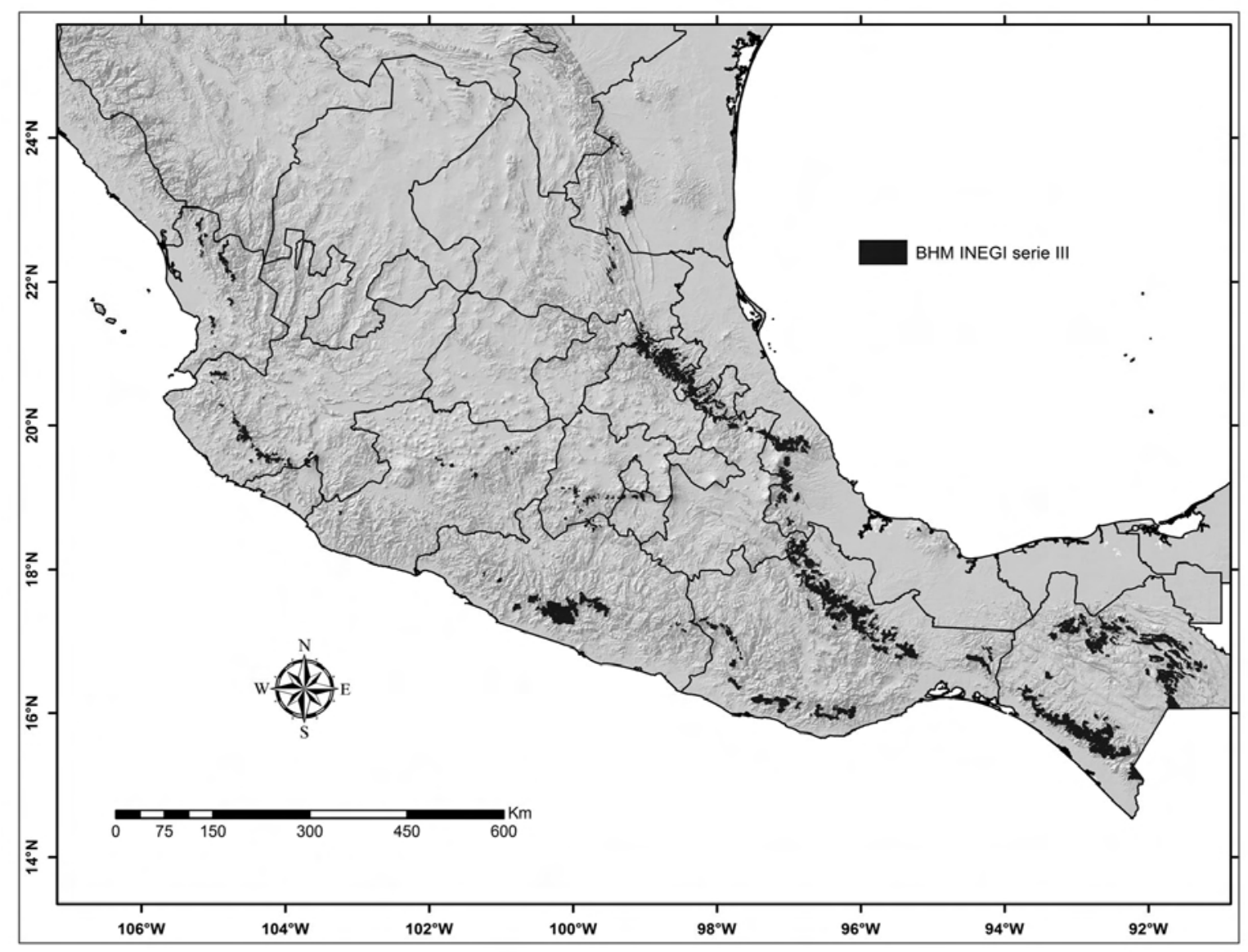

Fig. 1. Distribución geográfica actual del bosque húmedo de montaña (BHM) en México (tomado de INEGI, 2005). 
De todos los tipos de vegetación de México, el BHM es el ecosistema más amenazado y el tipo de vegetación que contiene la mayor riqueza de especies de plantas vasculares por unidad de superficie (Rzedowski, 1978, 1996; CONABIO, 2010; Villaseñor, 2010). A esta gran riqueza de especies asentada en una superficie de terreno montañoso relativamente pequeña, se le agrega una elevada heterogeneidad ambiental (clima, suelos y altitud) y un alto grado de fragmentación del hábitat en toda su área de distribución (Ramírez-Marcial et al., 2001; Vázquez-García, 1995). Recientemente, Villaseñor (2010) realizó una revisión exhaustiva de la literatura taxonómico-florística de este bioma y reporta que su riqueza florística en México asciende a 6,790 especies, pertenecientes a 1,625 géneros y 238 familias, más 665 variedades o subespecies. Se deben incluir, además, 271 especies introducidas pertenecientes a 207 géneros y 80 familias. La importancia de este bosque para la flora vascular de México, además de su gran riqueza y elevado porcentaje de endemismos, es extraordinaria ya que el BHM se distribuye en tan sólo el $0.6 \%$ de la superficie del país (ca. 11,719 $\mathrm{km}^{2}$ ), lo que significa que este tipo de vegetación contiene la más alta riqueza de especies y endemismos por unidad de superficie (Rzedowski, 1978, 1996; CONABIO, 2010; Villaseñor, 2010). En términos de protección, la situación del BHM es crítica, ya que se encuentra sujeto a una creciente presión humana, colocándolo en riesgo persistente y pone en tela de juicio la viabilidad de las estrategias de uso, manejo y conservación de su capital natural a escalas local, regional y nacional (Ramírez-Marcial et al., 2001; Ramírez-Marcial, 2002; CONABIO, 2010; Toledo-Aceves et al., 2010; González-Espinosa et al., 2011). Sin embargo, a pesar de la elevada riqueza de especies y de endemismos, en todos los trabajos publicados a la fecha, se da por sentado o se infiere que los atributos ambientales que limitan o promueven la distribución del BHM en México son relativamente bien conocidos. Los datos climáticos de distribución del BHM en México se han inferido a partir de los rasgos y tendencias registradas en las cartas climáticas existentes para el país o de las estaciones meteorológicas más cercanas a los sitios de estudio particulares. En consecuencia, a la fecha no se ha realizado un análisis objetivo que determine la relación existente entre los patrones de variabilidad espacial de la riqueza de plantas con las variables ambientales a través del área de distribución del BHM de México. Nuestra hipótesis es que la variación en la riqueza de especies del BHM tiene relación significativa con variables ambientales contemporáneas principalmente con el clima, las propiedades del suelo y la altitud. Este trabajo intenta responder la siguiente interrogante: ¿qué atributos ambientales contemporáneos podrían explicar la riqueza de especies de plantas del BHM de México? El objetivo fue analizar y determinar qué variables ambientales son las más importantes en la predicción de la variabilidad de la riqueza de especies que coexisten en el BHM de México.

\section{Materiales y Métodos}

Bases de datos. Especies.- El análisis incluye las especies conocidas del BHM, es decir aquellas especies que se han reportado en la literatura y tienen registro de herbario como especies distribuidas en el BHM de México. Con este criterio, el número de especies conocidas del BHM empleadas en el análisis fue de 6,790 (Villaseñor, 2010). Las especies discutidas en este trabajo son nativas de México; un taxón es considerado nativo si no existe evidencia publicada que indique su introducción al país. Por ello no se han incluido en los análisis alrededor de 271 especies consideradas como introducidas, la mayoría características del paisaje como malezas, tanto arvenses como ruderales, o conocidas solamente como plantas cultivadas. Se considera que las cifras presentadas son una buena aproximación sobre la diversidad del conocimiento florístico del BHM en el país.

Ambientales.- En el BHM en México prevalecen condiciones ambientales muy variables asociadas con el clima, la topografía, los suelos y el régimen hídrico. Estas condiciones varían de acuerdo al rango de distribución geográfica, pero principalmente en función de la latitud y la altitud. Las condiciones climáticas determinantes de este tipo de vegetación provienen de un número insuficiente de estaciones meteorológicas donde se distribuye el BHM, por lo que las determinantes climáticas de este tipo de vegetación deben considerarse estimaciones indirectas de las mismas. En este contexto, la mejor alternativa encontrada fue el empleo de la base de datos climática global WorldClim (<www.worldclim.org/current.html> consultado 21 de mayo, 2010), basada en la interpolación de datos climáticos conocidos de estaciones meteorológicas del mundo (Hijmans et al., 2005). El cuadro 1 muestra las variables ambientales consideradas en los análisis, que incluyen: (a) 19 variables bioclimáticas (BIO1-BIO19) obtenidas de WorldClim (Hijmans et al., 2005); (b) ocho variables de estacionalidad climática: precipitación de los meses húmedos, precipitación de los meses secos, temperatura media de los meses húmedos, temperatura media de los meses secos y cuatro variables de evapotranspiración: la evapotranspiración real anual (ETRA), la evapotranspiración real de los meses húmedos $\left(\right.$ ETRA $\left._{\mathrm{H}}\right)$, la evapotranspiración real de los meses secos $\left(\right.$ ETRA $\left._{\mathrm{s}}\right)$ y la proporción de la ETRA S $_{\mathrm{S}}$ ETRA $_{\mathrm{H}}$. La ETRA se calculó con base en el modelo de Turc (1954), según el cual,

$$
\mathrm{ETRA}=\mathrm{P} /\left[0.9+(\mathrm{P} / \mathrm{L})^{2}\right]^{1 / 2}
$$

donde $\mathrm{P}=$ precipitación total anual $(\mathrm{mm}), \mathrm{L}=300+25 \mathrm{~T}$ $+0.05 \mathrm{~T}^{3}$ y $\mathrm{T}=$ temperatura media anual $\left({ }^{\circ} \mathrm{C}\right)$; (c) siete variables edáficas: conductividad eléctrica, carbono orgánico, magnesio, materia orgánica, sodio, potasio y $\mathrm{pH}$. La información de los suelos proviene de 4,583 muestras tomadas a través del país (Ortiz-Solorio, 2002) y (d) un modelo digital de elevación (MDE) extraído de la base de datos GTOPO (<edc.usgs.gov/products/elevation/gtopo30/gtopo30.html>, consultado 25 de octubre, 2010). De cada propiedad del suelo 
Cuadro 1. Triada de variables ambientales consideradas en los análisis. Conjunto de variables bioclimáticas (a), de estacionalidad climática (b), propiedades de los suelos (c) y un modelo digital de elevación (d).

\begin{tabular}{|c|c|c|}
\hline \multicolumn{3}{|c|}{ a) Variables Bioclimáticas: } \\
\hline $\mathrm{BIO} 1$ & $=$ & Temperatura Media Anual \\
\hline $\mathrm{BIO} 2$ & $=$ & Rango promedio de temperaturas diarias $\left(\mathrm{T}_{\max }-\mathrm{T}_{\min }\right)$ \\
\hline $\mathrm{BIO} 3$ & $=$ & Isotermalidad (BIO2/BIO7)×100 \\
\hline $\mathrm{BIO} 4$ & $=$ & Estacionalidad de la temperatura (Desv. estand. $\times 100$ ) \\
\hline $\mathrm{BIO} 5$ & $=$ & Temperatura máxima del mes más cálido \\
\hline $\mathrm{BIO} 6$ & $=$ & Temperatura mínima del mes más frío \\
\hline $\mathrm{BIO} 7$ & $=$ & Rango anual de la temperatura (BIO5-BIO6) \\
\hline $\mathrm{BIO} 8$ & $=$ & Temperatura media del trimestre más húmedo \\
\hline $\mathrm{BIO} 9$ & $=$ & Temperatura media del trimestre más seco \\
\hline $\mathrm{BIO} 10$ & $=$ & Temperatura media del trimestre más cálido \\
\hline BIO 11 & $=$ & Temperatura media del trimestre más frío \\
\hline $\mathrm{BIO} 12$ & $=$ & Precipitación anual \\
\hline $\mathrm{BIO} 13$ & $=$ & Precipitación del mes más húmedo \\
\hline $\mathrm{BIO} 14$ & $=$ & Precipitación del mes más seco \\
\hline BIO 15 & $=$ & Estacionalidad de la precipitación \\
\hline BIO 16 & $=$ & Precipitación del trimestre más húmedo \\
\hline $\mathrm{BIO} 17$ & $=$ & Precipitación del trimestre más seco \\
\hline $\mathrm{BIO} 18$ & $=$ & Precipitación del trimestre más cálido \\
\hline BIO 19 & $=$ & Precipitación del trimestre más frío \\
\hline
\end{tabular}

\begin{tabular}{|c|c|c|}
\hline \multicolumn{3}{|c|}{ b) Variables de Estacionalidad Climática: } \\
\hline $\mathrm{PPH}$ & $=$ & $\begin{array}{l}\text { Precipitación Pluvial de la temporada lluviosa del año } \\
\text { (mayo a octubre) }\end{array}$ \\
\hline PPS & $=$ & $\begin{array}{l}\text { Precipitación Pluvial de la temporada seca del año } \\
\text { (noviembre a abril) }\end{array}$ \\
\hline $\mathrm{TH}$ & $=$ & $\begin{array}{l}\text { Temperatura promedio de la temporada lluviosa del } \\
\text { año (mayo a octubre) }\end{array}$ \\
\hline TS & $=$ & $\begin{array}{l}\text { Temperatura promedio de la temporada seca del año } \\
\text { (noviembre a abril) }\end{array}$ \\
\hline ETRA & $=$ & Evapotranspiración real anual \\
\hline ETRA $_{H}$ & $=$ & $\begin{array}{l}\text { Evapotranspiración real de la temporada lluviosa del } \\
\text { año (mayo a octubre) }\end{array}$ \\
\hline $\mathrm{ETRA}_{\mathrm{S}}$ & $=$ & $\begin{array}{l}\text { la evapotranspiración real de la temporada seca del } \\
\text { año (noviembre a abril) }\end{array}$ \\
\hline $\begin{array}{l}\text { ETRA }_{S} / \\
\text { ETRA }_{H}\end{array}$ & $=$ & Proporción de la ETRA $_{S}$ y ETRA $_{H}$ \\
\hline
\end{tabular}

\begin{tabular}{ll}
\hline c) Propiedades de los Suelos: \\
\hline $\mathrm{pH}$ & $=$ Potencial de Hidrógeno \\
$\mathrm{CE}$ & $=$ Conductividad Eléctrica \\
$\mathrm{CO}$ & $=$ Carbono Orgánico \\
$\mathrm{MO}$ & $=$ Materia Orgánica \\
$\mathrm{Mg}$ & $=$ Magnesio \\
$\mathrm{Na}$ & $=$ Sodio \\
$\mathrm{K}$ & $=$ Potasio
\end{tabular}

d) Modelo Digital de Elevación (altitud):

MDE = altitud se generó una capa ambiental mediante interpolación usando el inverso de la distancia ponderada implementado en IDRISI Andes $^{\mathrm{TM}}$ (Eastman, 2006), con tamaño de píxel de 30 segundos de arco, que equivale a $1 \mathrm{~km}^{2}$ aproximadamente.

Espaciales.- El territorio continental de México se dividió en 249 celdas de $1^{\circ}$ de latitud y longitud, referidas a un sistema de coordenadas geodésicas entre $14^{\circ}$ y $32^{\circ}$ de latitud norte y $86^{\circ}$ a $117^{\circ}$ de longitud oeste. Con base en la localización geográfica, las especies de plantas registradas para el BHM fueron asignadas a una celda en particular. El número total de celdas con presencia de BHM fueron 61. Las capas de variables climáticas, edáficas y altitudinal fueron recortadas sobreponiendo la retícula de las 61 celdas con BHM mediante el uso de ArcMap (ESRI, 2010).

El número total de variables incluidas en los análisis fue de 36 y la amplitud de la variabilidad ambiental contenida en las 61 celdas que pudiesen explicar la mayor variación en la riqueza de especies, se cuantificó con el cálculo de sus estadísticas descriptivas (mínimo, máximo, rango, media, desviación estándar y el coeficiente de variación) mediante el empleo de IDRISI 15.0 Andes (Eastman, 2006). En total se obtuvieron 216 parámetros ambientales, los cuales fueron transformados para mejorarlos o ajustarlos a una distribución normal. Las variables se transformaron con $\log _{10}(\mathrm{x})$ o $\log _{10}(\mathrm{x}+1)$ si el valor de $\mathrm{x}$ era bajo o cercano a cero, mientras que aquellos parámetros expresados en porcentajes o proporciones se transfomaron con arcoseno $\left(\mathrm{x}^{1 / 2}\right)($ Zar, 1999).

Análisis estadísticos. Se construyó una matriz de datos con los atributos físicos y ambientales y la riqueza de especies por celda. Primero se hicieron predicciones con base en la estimación de los parámetros usando modelos generales lineales, GLM (SAS, 2008). Estos modelos no toman en cuenta la estructura geográfica espacial de los datos excepto aquella estructura implícita en las variables predictivas (Kreft y Jetz, 2007). La autocorrelación espacial de la riqueza de especies y las variables predictivas es una característica intrínseca en los conjuntos de datos macroecológicos (Legendre, 1993); ésta infla los errores tipo I de la estadística tradicional y puede afectar los parámetros estimados (Lennon, 2000). La detección de autocorrelación de las variables predictoras se evaluó mediante regresión múltiple con el método stepwise y la opción factores de inflación de la varianza (vif) implementadas en SAS (2008). De acuerdo con Der y Everitt (2002), las variables cuyos vif fueron $\geq$ 10.0 se eliminaron del análisis. El análisis espacial se realizó mediante el software Spatial Analysis in Macroecology, SAM (Rangel et al., 2006). La selección del mejor modelo de ajuste se basó en la reducción de autocorrelación espacial en los residuales (Legendre y Legendre, 1998) y en la minimización de los valores del Criterio de Información Akaike (AICc). De acuerdo con Burnham y Anderson (2002), el modelo con el valor más bajo de AICc es considerado el 
mejor modelo. Se empleó la estadística $I$ de Moran implementada en SAM (Rangel et al., 2006) para detectar autocorrelación espacial en los residuales del modelo. El valor esperado del índice $I$ de Moran carente de autocorrelación espacial debe ser cercano a cero (Fortín y Dale, 2005). El modelo final se seleccionó con base en el valor más bajo de AICc, con las variables predictivas libres de autocorrelación espacial, con valores del índice $I$ de Moran estadísticamente iguales a cero a cualquier distancia, con número condicional $\mathrm{CN}<5$ y valores de VIF < 10 (Der y Everitt, 2002; SAS, 2008).

\section{Resultados}

En el BHM de México existen zonas amplias de tamaño variable que presentan tanto elevada riqueza de especies como otras que contienen un bajo número de especies. Las zonas de BHM que presentan las concentraciones más elevadas de riqueza de especies se localizan en el sureste del país, en los estados de Chiapas y Oaxaca, pero destacan también sitios en Guerrero, Jalisco y la parte central y sur de Veracruz, así como sus límites con Puebla e Hidalgo. En estos estados la riqueza es mayor a 1,500 especies, pero tanto Chiapas como Oaxaca albergan zonas con más 2,000 especies, con un mínimo de 2,006 en las montañas del occidente de Chiapas con los límites con Oaxaca y un máximo de 2,324 especies en las montañas del norte de Oaxaca. A lo largo de toda la Faja Volcánica Transmexicana (Ferrusquía-Villafranca, 1993), la riqueza de especies del BHM se mantiene principalmente entre 1,130 y 1,810 especies pero en sus extremos occidental y oriental la riqueza alcanza hasta 2,065 y 2,181 , respectivamente. Existen zonas con una riqueza relativamente baja (500-750) localizadas a barlovento de la planicie costera del Pacífico, en Chiapas y sur de Oaxaca y Michoacán, así como en la Sierra Madre Occidental en Durango y Sonora. Otras áreas con una riqueza de especies moderada (760970) ocurren en la Sierra Madre del Sur en Oaxaca y límites con Veracruz, en los límites de Guerrero-Oaxaca y sur de Puebla, así como en Tamaulipas y en los límites de Durango y Nayarit. Las zonas de baja riqueza de especies están localizadas a barlovento de la Sierra Madre Occidental, en los límites entre Jalisco y Nayarit, y en las montañas de la Sierra Madre Occidental en Durango y Sinaloa; sobre la Sierra Madre Oriental se ubican en San Luis Potosí, Tamaulipas y Nuevo León.

La amplitud de variación en la riqueza de especies del BHM a través de su ámbito de distribución conocida en México se explica en función de algunas variables ambientales implícitas en el clima, el suelo y el modelo digital de elevación. El cuadro 2 muestra los resultados combinados de los análisis de regresión lineal ordinaria, OLS (Rangel et al., 2010) y de regresión múltiple (SAS, 2008). El modelo más parsimonioso (con el valor más bajo de $\mathrm{AIC}_{\mathrm{C}}$ ) seleccionó a cinco variables ambientales, las cuales explican $58.6 \%$
$(P<0.0001, \mathrm{n}=59)$ del total de la variación en la riqueza de especies (Cuadro 2). El orden de importancia de las variables seleccionadas, proporcionado por los coeficientes de determinación parcial $\left(R_{\text {parcial }}^{2}\right)$, fueron: el rango de precipitación pluvial en los meses húmedos del año $\left(\mathrm{PPH}_{\mathrm{rnk}}\right)$, el modelo digital de elevación (MDE), el valor más bajo de la evapotranspiración anual (ETRA ${ }_{\text {min }}$ ), la variación en la estacionalidad de la precipitación pluvial (BIO15) y el rango de la concentración del Carbono Orgánico $\left(\mathrm{CO}_{\mathrm{rnk}}\right)$ en el suelo (Cuadro 2). La magnitud de importancia jerárquica de las dos primeras variables contienen los valores predictivos más altos $\left(18.9>R_{\text {parcial }}^{2} \leq 21.2\right)$; mientras que el resto de las variables contienen un valor predictivo relativamente bajo $\left(3.5>R_{\text {parcial }}^{2} \leq 7.5\right)$. Los valores tanto del $\mathrm{AIC}_{\mathrm{C}}$, el CN y los VIF $<10$ sugieren que la inflación de los errores tipo I difícilmente podrían alterar las predicciones de los parámetros estimados (Legendre, 1993; Der y Everitt, 2002; Rangel et

Cuadro 2. Coeficientes de determinación $\left(R^{2}\right)$ y contribución parcial $\left(R_{\text {parcial }}^{2}\right)$ de las variables ambientales predictivas (VAP) y porcentaje de la varianza explicada de la riqueza de especies restringidas al Bosque Húmedo de Montaña de México. Los valores de las estadísticas $R^{2}$, F, P, AICC, CN y VIF son derivados del modelo de regresión lineal obtenido con SAM y SAS.

\begin{tabular}{|c|c|c|c|c|c|c|c|}
\hline $\begin{array}{c}\text { Variables } \\
\text { ambientales }\end{array}$ & $R^{2}$ & $R_{\text {parcial }}^{2}$ & $\mathrm{~F}$ & $P$ & $\mathrm{AIC}_{\mathrm{C}}$ & $\mathrm{CN}$ & VIF \\
\hline $\begin{array}{l}\text { Modelo } \\
\text { completo }\end{array}$ & 0.5858 & - & 14.99 & $<0.0001$ & 880.5 & 2.74 & - \\
\hline $\mathrm{PPH}_{\mathrm{rnk}}$ & & 0.2119 & 15.33 & 0.0002 & & & 1.1418 \\
\hline $\mathrm{MDE}_{\text {avg }}$ & & 0.1894 & 17.72 & $<0.0001$ & & & 2.3265 \\
\hline $\mathrm{ETRA}_{\text {min }}$ & & 0.0750 & 7.88 & 0.0069 & & & 2.1147 \\
\hline $\mathrm{BIO} 15_{\mathrm{de}}$ & & 0.0736 & 8.83 & 0.0044 & & & 1.0709 \\
\hline $\mathrm{CO}_{\mathrm{rnk}}$ & & 0.0359 & 4.59 & 0.0368 & & & 1.0833 \\
\hline
\end{tabular}

al., 2006, 2010).

Las variables más importantes en este modelo fueron la precipitación pluvial de los meses húmedos del año (mayo a octubre) y la altitud, las cuales conjuntamente explican $40 \%$ de la variación en la riqueza de especies. Ambas variables presentan una amplia variabilidad a través de la distribución del BHM (Figura 2A, B); por ejemplo, el rango de la PPH fluctúa de $376 \mathrm{~mm}$ a $3,394 \mathrm{~mm}$, con un promedio de 1,383 $\mathrm{mm}$ y un coeficiente de variación de $32 \%$. Por su parte la variabilidad de la distribución altitudinal (MDE) del BHM fluctúa desde 216 m s.n.m. hasta 3,342 m s.n.m., con una media de 1,691 m s.n.m. y un coeficiente de variación de $37 \%$. En particular, en el rango de la precipitación pluvial $\left(\mathrm{PPH}_{\mathrm{rnk}}\right)$ durante los meses lluviosos del año (mayo-octubre) con presencia de BHM equivale a aproximadamente el $84 \%$ del total anual. Por otra parte, tanto el valor mínimo extremo de la evapotranspiración real anual (ETRA $\left.{ }_{\text {min }}\right)$, como la estacionalidad de la precipitación (BIO15 de, contribuyeron a explicar la variación en la riqueza de especies con porcenta- 

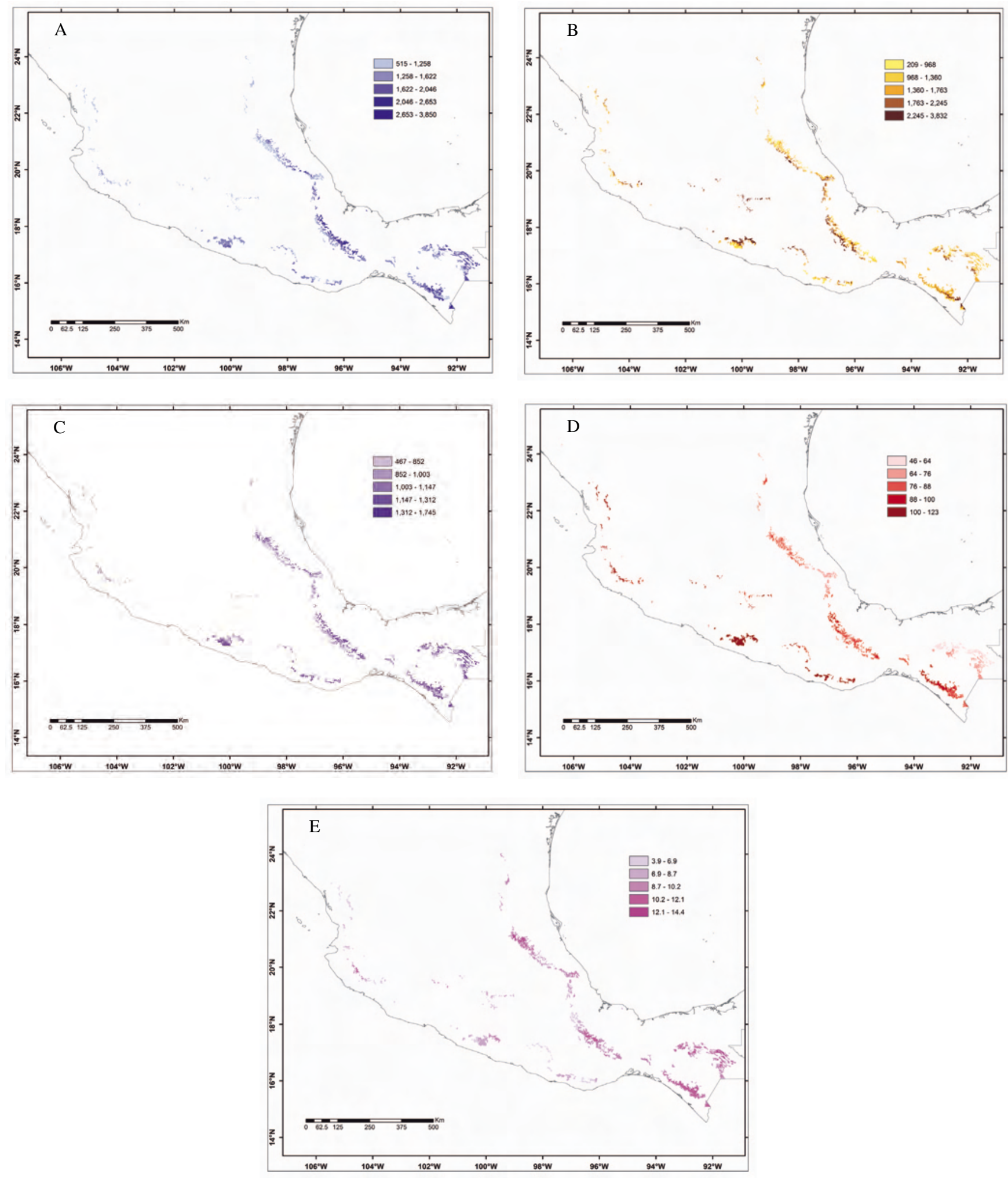

Fig. 2. A) Precipitación pluvial de los meses húmedos del año; B) modelo digital de elevación (altitud) en m s.n.m.; C) evapotranspiración real anual; D) estacionalidad de la precipitación; E) carbono orgánico en el suelo. 
jes relativamente bajos (7.5 y 7.4\%, respectivamente; Figura 2C, D). La evapotranspiración real anual (ETRA) combina la temperatura media y la precipitación total anual, mientras que la estacionalidad de la precipitación (BIO15) es una expresión de la variabilidad en la precipitación total anual, por lo que ambas son expresiones de energía. En cambio, el rango de la variación del Carbono Orgánico en el suelo $\left(\mathrm{CO}_{\text {rnk }}\right)$, contribuyó a explicar tan solo un $3.6 \%$ de la riqueza de especies del BHM (Figura 2E). El Carbono Orgánico es una propiedad intrínseca del suelo que expresa su fertilidad y por tanto su calidad. Las variables de estacionalidad climática (PPH, ETRA) y bioclimática (BIO15) tomadas juntas, explican el $36 \%$ de la variabilidad en la riqueza de especies, seguidas de la altitud (MDE) con un $18.9 \%$ y en menor medida por la fertilidad (CO) de los suelos que sostienen la vegetación del BHM.

\section{Discusión}

Con excepción de la altitud y el carbono orgánico, las variables seleccionadas por el modelo fueron variables de energía (PPH, ETRA y BIO15) o de agua-energía. La hipótesis de que la energía se correlaciona positivamente con la riqueza de especies de plantas, ha sido sustentada por estudios a escala global (Francis y Currie, 2003; Kreft y Jetz, 2007) y para formas de crecimiento arbóreo a escala regional $\mathrm{Cu}$ rrie y Paquin, 1987; O'Brien et. al., 1998, 2000) y del paisaje (González-Espinosa et al., 2004). En el análisis global de Francis y Currie (2003) se presentó una relación entre la riqueza de familias de Angiospermas y la temperatura promedio anual, la evapotranspiración potencial y la precipitación total anual. De acuerdo con estos autores, los patrones de riqueza de especies dependen principalmente de la disponibilidad simultánea de agua y calor. Los modelos de Francis y Currie (2003) indican que la forma de la relación riqueza-calor depende de la disponibilidad de agua y que la relación riqueza-agua depende del calor. Por otra parte, el análisis global de Kreft y Jetz (2007) sobre los determinantes ambientales e históricos de los patrones en riqueza indican que la evapotranspiración potencial global, el número de días húmedos por año y la heterogeneidad topográfica y del hábitat son los predictores más importantes de la riqueza de especies de plantas vasculares.

En esta investigación, la evaporación real anual resultó seleccionada en tercer lugar en el modelo para explicar la riqueza de especies en el BHM, precedida por la precipitación en los meses húmedos del año y la altitud. Este resultado concuerda con los hallazgos de Currie y Paquin (1987) a escala regional y con los de González-Espinosa et al. (2004) a escala del paisaje en Chiapas. Estos autores encontraron una asociación positiva entre la evapotranspiración real anual y la riqueza de especies de árboles a ambas escalas de resolución espacial (sensu Whittaker et al., 2003). Currie y Paquin (1987) reportaron un modelo logístico que explicó más del
$76 \%$ de la varianza en la riqueza de árboles en Norteamérica en función de la evapotranspiración real anual. A escala del paisaje, González-Espinosa et al. (2004) encontraron una relación curvilinear que explicó $41.4 \%$ de la varianza en la diversidad de árboles en Chiapas. Los predictores de la diversidad de árboles en Chiapas fueron la evapotranspiración total anual, la estacionalidad de la evapotranspiración durante los meses secos del año y la fertilidad-calidad de los suelos (González-Espinosa et al., 2004).

En México, la precipitación pluvial varía enormemente en espacio y tiempo (García, 1965); es heterogénea a lo largo del año y geográficamente aumenta en dirección norte-sur debido a la influencia de la latitud. Tal variabilidad está dada por la influencia del océano Pacífico y el Golfo de México, por la orografía del país, los rasgos de circulación atmosférica (García, 1965) y por la conectividad del fenómeno del Niño (Cavazos y Hastenrath, 1990). La distribución de la precipitación en México ha sido ampliamente documentada por García (1965) y por Mociño y García (1974), quienes señalan que aproximadamente $70 \%$ de la precipitación pluvial en México se registra durante los meses de mayo a octubre. En particular, la presencia del BHM sobre las principales cadenas montañosas del país está influenciada por la presencia de nubes o neblina de altitud variable (Rzedowski, 1996; Challenger, 1998; Luna-Vega et al., 2006; Villaseñor, 2010). Este fenómeno atmosférico es conocido como "efecto Massenerhebung" postulado por Grubb (1971), "mountain mass elevation effect" o "efecto de masa de alta montaña" (Flenley, 1995). La región del BHM más lluviosa se encuentra al sur del paralelo $17^{\circ} \mathrm{N}$ y comprende las pendientes montañosas del centro y sur del país que se inclinan al Golfo de México. El barlovento de esta región está directamente expuesto a los vientos húmedos del Golfo de México asociados con los vientos alisios, los nortes y los ciclones tropicales. Durante el verano, en las montañas dominan los vientos alisios con dirección este a oeste, los cuales recogen gran cantidad de humedad de las aguas calientes del Golfo de México. Los vientos alisios encuentran las laderas montañosas a barlovento, provocando que éstos asciendan, se enfríen adiabáticamente, se condensen y precipiten su abundante humedad en forma de lluvia. La cantidad de lluvia descargada a barlovento se incrementa considerablemente por la presencia de ciclones provenientes del Mar de las Antillas, los cuales son más frecuentes en los meses de septiembre y octubre (García, 1965; Mociño y García, 1974).

Sobre la vertiente del Golfo hay cuatro áreas con precipitación mayor de 3,500 $\mathrm{mm}$ al año: (1) la región al norte del paralelo $20^{\circ} \mathrm{N}$ en las sierras de Teziutlán y Zacapoaxtla, (2) la situada al sur del paralelo $18^{\circ} \mathrm{N}$ en las Sierras de Ixtlán, Mixes y La Chinantla, (3) la Sierra de los Tuxtlas y (4) la vertiente norte de las montañas del norte de Chiapas. El máximo de la precipitación en estas áreas se presenta al norte de Oaxaca en La Chinantla, en altitudes entre 1,730 
y 1,450 m s.n.m., con una precipitación anual que fluctúa entre 5,500 $\mathrm{mm}$ y $5,800 \mathrm{~mm}$ respectivamente. La Chinantla es la región más lluviosa en toda la amplitud de distribución del BHM, por lo que constituye la región hiper-húmeda de esta comunidad en México (Meave et al., 2006).

Sobre las montañas que presentan BHM, la precipitación es más abundante en las montañas del Golfo que en las del Pacífico, pues las del Pacífico no reciben más de 2,000 mm y eso en sitios aislados. Hay, sin embargo, otra zona de lluvia muy abundante, con precipitación mayor de 3,500 mm localizada sobre la porción sureste de la Sierra Madre de Chiapas. En esta zona, la elevada precipitación está fuertemente influenciada por la ocurrencia de los ciclones tropicales tanto del Pacífico como del Golfo de México, con un máximo en septiembre. En el resto de la vertiente del Pacífico, la precipitación ocurre principalmente en verano y parte del otoño; durante el verano la mayor parte de la precipitación proviene del océano Pacífico, mientras que la del otoño está fuertemente influenciada por los ciclones tropicales, con un aumento de la precipitación en septiembre. Es notorio también que a principios del otoño la precipitación se incremente sobre la vertiente sur de la Faja Volcánica Transmexicana por la influencia de los ciclones tropicales, tanto del Golfo como del Pacífico. A lo largo de esta región se presentan de 1,200 a 1,500 mm de lluvia al año, con su máximo en septiembre, lo que pone de manifiesto la influencia de los ciclones.

Por otro lado, los bosques húmedos de montaña de México y Centroamérica acumulan grandes cantidades de materia orgánica en el suelo (Clark et al., 2000; Velázquez et al., 2000), siendo el carbono orgánico su principal elemento. Su elevada acumulación depende de las bajas tasas de descomposición debido a temperaturas relativamente bajas, a las condiciones de humedad de los suelos (Jenny, 1980) y a la baja calidad de la hojarasca con elevados contenidos de lignina y polifenoles y bajas proporciones de N:C y P:C (Clark et al., 2000). La débil relación entre la riqueza de especies y el carbono orgánico del suelo sugiere la necesidad de realizar estudios de los procesos edáficos involucrados en la acumulación de carbono orgánico a través del rango de distribución del BHM de México.

\section{Conclusiones}

En el BHM de México existe una relación significativa entre la riqueza de especies con la precipitación pluvial en los meses húmedos del año y la evapotranspiración real anual, como una expresión categórica de la relación agua-energía. Ambas variables son representaciones sustitutas de la humedad relativa en el ámbito de distribución del BHM en México. La variabilidad en la riqueza de especies es consistente con las predicciones de las hipótesis especies-energía y de la dinámica agua-energía, formalizadas por Wright (1983) y examinadas por varios autores a escalas global (Francis y
Currie, 2003; Kreft y Jetz, 2007), regional (O'Brien et al., 1998, 2000) y del paisaje (González-Espinosa et al., 2004). Además, los resultados indican que la explicación de la variabilidad en la riqueza del BHM está asociada con variables ambientales en forma de agua-energía y de las propiedades de los suelos que sostienen este bioma con una de las mayores riquezas de plantas vasculares de México. Sin embargo, a pesar de que la variación en la riqueza de especies se explicó significativamente con pocas variables, otro porcentaje de la riqueza permaneció no explicado. Esto sugiere que las variables ambientales del clima contemporáneo no son los promotores exclusivos que mantienen la riqueza de especies de plantas vasculares distribuidas en el BHM de México, pero si son factores significativamente importantes correlacionados con la variabilidad de la riqueza de especies observada.

Resultó evidente que la distribución geográfica de la precipitación pluvial durante los meses húmedos del año a través del área de distribución del BHM de México está íntimamente ligada con la orografía (p. ej., con la altitud de las montañas del país) así como con la distribución de los rasgos más prominentes de la circulación atmosférica. Las montañas actúan como barreras donde se descarga gran parte de la humedad acarreada por los vientos de las capas bajas de la atmósfera, haciendo que aumente la cantidad de lluvia en sus vertientes expuestas a vientos húmedos, así como en sus partes más elevadas del continente, por ascenso y enfriamiento adiabático del aire. Los ciclones tropicales tanto del Golfo de México como del océano Pacífico tienen una gran influencia en los máximos de precipitación de los lugares situados en sus vertientes, pues los máximos coinciden con la época de mayor frecuencia de dichas perturbaciones atmosféricas. La riqueza de especies de plantas vasculares que se distribuyen en el BHM de México tiene explicación correlativa con las variables de estacionalidad climática contemporáneas, con la altitud y con la acumulación de carbono orgánico en los suelos. La acumulación de carbono orgánico en los suelos del BHM amerita un análisis profundo ya que éstos sustentan al bioma con mayor riqueza de especies de plantas vasculares de todos los tipos de vegetación de México.

\section{Agradecimientos}

El primer autor agradece al Consejo Nacional de Ciencia y Tecnología el apoyo para realizar una estancia sabática en el Instituto de Biología de la Universidad Nacional Autónoma de México. Mi agradecimiento también para Tila María Pérez Ortíz, Directora del Instituto de Biología y para Claudio Delgadillo Moya, Jefe del Departamento de Botánica, ambos de esta misma institución, por todas las facilidades proporcionadas durante la realización de mi año sabático. Agradecemos la amabilidad y gentileza del personal de la biblioteca del Instituto de Biología por su apoyo incondicional en la búsqueda de material bibliográfico. 


\section{Literatura citada}

Bruijnzeel L.A., Waterloo M.J., Proctor J., Kuiters A.T. y Kotterink B. 1993. Hydrological observations in montane rain forests on Gunung Silam, Sabah, Malaysia, with special reference to the 'Massenerhebung' effect. Journal of Ecology 81:141-167.

Burnham K.P. y Anderson D.R. 2002. Model Selection and Multimodel Inference: A Practical Information-Theoretic Approach. Springer, Nueva York.

Cavazos T. y Hastenrath S. 1990. Convection and rainfall over Mexico and their modulation by the southern oscillation. International Journal of Climatology 10:377-386.

Challenger A. 1998. Utilización y Conservación de los Ecosistemas Terrestres de México, Pasado, Presente y Futuro. Comisión Nacional para el Conocimiento y Uso de la Biodiversidad, México, D.F.

Churchill S.P., Balslev H., Forero E. y Luteyn J.L. 1995. Biodiversity and Conservation of Neotropical Montane Forests. The New York Botanical Garden, Nueva York.

Clark K.L., Lawton R.O. y Butler P.R. 2000. The physical environment. En: Nadkarni N.M. y Wheelwright N.T. Eds. Monteverde, Ecology and Conservation of a Tropical Cloud Forest, pp. 1538, Oxford University Press, Nueva York.

CONABIO [Comisión Nacional para el Conocimiento y Uso de la Biodiversidad]. 2010. El Bosque Mesófilo de Montaña en México: Amenazas y Oportunidades para su Conservación y Manejo Sostenible. Comisión Nacional para el Conocimiento y Uso de la Biodiversidad, México, D.F.

Currie D.J. y Paquin V. 1987. Large-scale biogeographical patterns of species richness of trees. Nature 329:326-327.

Der G. y Everitt B.S. 2002. A Handbook of Statistical Analyses using SAS. Chapman and Hall/CRC, Boca Raton.

Eastman J.R. 2006. IDRISI 15.0 Andes: Guide to GIS and Image Processing. Clark Labs, Clark University, Worcester.

ESRI [Environmental Systems Research Institute]. 2010. ArcGIS Desktop 10. Environmental Systems Research Institute, Redlands.

Ferrusquía-Villafranca I. 1993. Geology of Mexico: a synopsis. En: Ramamoorthy T.P., Bye R., Lot A. y Fa J. Eds. Biological Diversity of Mexico: Origins and Distribution, pp. 3-107, Oxford University Press, Nueva York.

Flenley J.R. 1995. Cloud forest, the Massenerhebung effect, and ultraviolet insolation. En: Hamilton L.S., Juvik J.O. y Scatena F.N. Eds. Tropical Montane Cloud Forests. Ecological Studies 110, pp 150-155, Springer-Verlag, Nueva York.

Fortin M.J. y Dale M.R.T. 2005. Spatial Analysis: A Guide for Ecologists. Cambridge University Press, Cambridge.

Francis A.P. y Currie D.J. 2003. A globally consistent richnessclimate relationship for Angiosperms. The American Naturalist 161:523-536.

García E. 1965. Distribución de la precipitación en la República Mexicana. Publicaciones del Instituto de Geografía 1:171-191.

González-Espinosa M., Meave J.A., Lorea-Hernández F.G., Ibarra-Manríquez G. y Newton, A.C. Eds. 2011. The red list of Mexican Cloud Forest trees. Fauna \& Flora International, Cambridge. Disponible en línea: <http://globaltrees.org/rl_mexican_cloudforest.html>

González-Espinosa M., Rey-Benayas J.M., Ramírez-Marcial N., Huston M.A. y Golicher D. 2004. Tree diversity in the northern
Neotropics: regional patterns in highly diverse Chiapas, Mexico. Ecography 27:741-756.

Grubb P.J. 1971. Interpretation of the 'Massenerhebung effect' on tropical mountains. Nature 229:44-45.

Hamilton L.S., Juvik J.O. y Scatena F.N. Eds. 1995. Tropical Montane Cloud Forests. Ecological Studies 110, Springer-Verlag, Nueva York.

Hijmans R.J., Cameron S.E., Parra J.L., Jones P.G. y Jarvis A. 2005. Very high resolution interpolated climate surfaces for global land areas. International Journal of Climatology 25:1965-1978.

Huber O. Ed. 1986. La Selva Nublada de Rancho Grande Parque Nacional "Henry Pittier": El Ambiente Físico, Ecología Vegetal y Anatomía Vegetal. Fondo Editorial Acta Científica Venezolana/Seguros Anauco C.A., Caracas.

INEGI [Instituto Nacional de Estadística y Geografía]. 2005. Carta de uso del suelo y vegetación. Serie III. Escala 1:250,000. Instituto Nacional de Estadística y Geografía. México, Aguascalientes.

Jenny H. 1980. The Soil Resource: Origin and Behavior. SpringerVerlang, Nueva York.

Kappelle M. y Brown A.D. Eds. 2001. Los Bosques Nublados del Neotrópico. World Conservation Union/Instituto Nacional de Biodiversidad, Santo Domingo de Heredia.

Kreft H. y Jetz W. 2007. Global patterns and determinants of vascular plant diversity. Proceedings of the National Academy of Science of the United States of America 104:5925-5930.

Legendre P. 1993. Spatial autocorrelation: trouble or new paradigm. Ecology 74:1659-1673.

Legendre P. y Legendre L. 1998. Numerical Ecology. Elsevier. Amsterdam.

Lennon J.J. 2000. Red-shifts and red herrings in geographical ecology. Ecography 23:101-113.

Luna-Vega I., Alcántara-Ayala O., Ruíz-Jiménez C.A. y ContrerasMedina R. 2006. Composition and structure of humid montane oak forests at different sites in central and eastern Mexico. En: Kappelle M. Ed. Ecology and Conservation of Neotropical Montane Oak, pp. 101-112, Springer-Verlag, Berlín.

Meave J.A., Rincón A. y Romero-Romero M.A. 2006. Oak forests of the hyper-humid region of La Chinantla, northern Oaxaca range, Mexico. En: Kappelle M. Ed. Ecology and Conservation of Neotropical Montane Oak Forests, pp. 113-125, SpringerVerlag, Berlín.

Mociño P.A. y García E. 1974. The climate of Mexico. En: Byrson R.A. y Hare F.K. Eds. Climates of North America, pp. 345-404, Elsevier, Londres.

O’Brien E.M., Whittaker R.J. y Field R. 1998. Climate and woody plant diversity in southern Africa: relationships at species, genus and family levels. Ecography 21:495-509.

O`Brien E.M., Field R. y Whittaker R.J. 2000. Climatic gradients in woody plant (tree and shrub) diversity: water-energy dynamics, residual variation, and topography. Oikos 89:588-600.

Ortiz-Solorio C. 2002. Evaluación de la degradación del suelo causada por el hombre en la República Mexicana, escala 1:250 000. Secretaría del Medio Ambiente y Recursos Naturales/Colegio de Postgraduados, Montecillo.

Ramírez-Marcial N., González-Espinosa M. y Williams-Linera G. 2001. Anthropogenic disturbance and tree diversity in montane rain forests in Chiapas, Mexico. Forest Ecology and Management 154:311-326.

Ramírez-Marcial N. 2002. Disturbio humano y la diversidad de ár- 
boles y arbustos del Bosque Mesófilo en las montañas del norte de Chiapas. Tesis doctoral, Instituto de Ecología A.C., Xalapa. $158 \mathrm{pp}$.

Rangel T.F.L.V.B., Diniz-Filho J.A.F. y Bini L.M. 2006. Toward an integrated computational tool for spatial analysis in macroecology and biogeography. Global Ecology and Biogeography 15:321-327.

Rangel T.F., Diniz-Filho J.A.F. y Bini L.M. 2010. SAM: a comprehensive application for spatial analysis in macroecology. Ecography 33:46-50.

Rzedowski J. 1978. Vegetación de México. Limusa, México, D.F.

Rzedowski J. 1996. Análisis preliminar de la flora vascular de los bosques mesófilos de montaña de México. Acta Botanica Mexicana 35:25-44.

SAS Institute. 2008. Statistical Analysis System 9.2. SAS Institute Inc., Cary.

Stadtmüller T. 1987. Cloud Forests in the Humid Tropics. A Bibliographic Review. United Nations University/Centro Agronómico Tropical de Investigación y Enseñanza, Turrialba.

Toledo-Aceves T., Meave J.A., González-Espinosa M. y Ramírez-Marcial N. 2010. Tropical montane cloud forests: current threats and opportunities for their conservation and sustainable management in Mexico. Journal of Environmental Management 92:974-981.

Turc L. 1954. Le bilan d'eau des sols: relations entre les preci- pitation, l'évaporation et l'écoulement. Annales Agronomiques 5:491-596.

Vázquez-García J.A. 1995. Cloud forest archipelagos: Preservation of fragmented montane ecosystems in tropical America. En: Hamilton L.S., Juvik J.O. y Scatena F.N. Eds. Tropical Montane Cloud Forests, pp. 315-332, Springer-Verlag, Nueva York.

Velázquez A., Toledo V.M. y Luna I. 2000. Mexican temperate vegetation. En: Barbour M.G. y Billings W.D. Eds. North American Terrestrial Vegetation, pp. 573-592, Cambridge University Press, Cambridge.

Villaseñor J.L. 2010. El Bosque Húmedo de Montaña en México y sus Plantas Vasculares: Catálogo Florístico-Taxonómico. Comisión Nacional para el Conocimiento y Uso de la Biodiversidad, México, D.F.

Vogelmann H.M. 1973. Fog precipitation in the cloud forests of Eastern Mexico. BioScience 23:96-100.

Whittaker R.J., Willis K.J., y Field R. 2003. Climatic-energetic explanations of diversity: a macroscopic perspective. En: Blackburn T.M. y Gaston K.J. Eds. Macroecology, Concepts and Consequences, pp. 107-129, Blackwell Science Ltd., Oxford.

Wright D.H. 1983. Species-energy theory: an extension of speciesarea theory. Oikos 41:496-506.

Zar J.H. 1999. Biostatistical Analysis. Prentice Hall. Englewood Cliffs.

Recibido: 7 de junio de 2011

Aceptado: 9 de octubre de 2011 